\title{
On ergodic properties of restrictions of inner functions
}

\author{
N. F. G. MARTIN \\ Department of Mathematics, Math-Astronomy Building, University of Virginia, \\ Charlottesville, VA 22903, USA
}

(Received 22 November 1986 and revised 16 July 1987)

\begin{abstract}
We consider inner functions on the unit disk which have a finite number of singularities on the unit circle. The restriction of such functions to the circle are maps onto the circle. We give sufficient conditions that these restrictions are exact endomorphisms whose natural extensions are Bernoulli and that the entropy is given by Rohlin's formula, $h(f)=\int \log \left|f^{\prime}\right| d \mu$. We also give the entropy in closed form if $f^{\prime}$ is in the Nevalinna class $N$. An example is considered. In the last section we show that if two restrictions are metrically isomorphic, they are diffeomorphic.
\end{abstract}

\section{Introduction}

We consider restrictions to the unit circle of inner functions on the unit disk which have a finite nonempty set of singularities on the circle. In an earlier paper [11] we studied the maps which were continuous on the circle and showed they were exact endomorphisms whose natural extensions are Bernoulli if their derivative on the circle was larger than one in absolute value.

In $\S 2$ we use the results of Rychlik [19] to give a sufficient condition that restrictions of inner functions with a finite nonempty set of singularities are exact endomorphisms whose natural extensions are Bernoulli. In $\S 3$ we give sufficient conditions that the entropy of such restrictions is given by Rohlin's formula, $h(f)=\int \log \left|f^{\prime}\right| d \mu$. If $f^{\prime}$ is in the Nevalinna class $N$ we evaluate this integral in terms of the critical points of $f$ in $\mathbb{O}$ and the fixed point of $f$ in $\mathbb{O}$. In $\S 4$ an example is considered, and in $\S 5$ we show that metrically isomorphic restrictions are conformally conjugate to within a rotation.

The study of ergodic properties of inner functions was begun by Aaronson [1], [2] and Neuwirth [14], although Adler and Kemperman had studied special inner functions prior to these papers. Subsequent work can be found in $[7,11,12,16,17,20]$.

The author would like to thank François Ledrappier, Barbara MacCluer, and Loren Pitt for useful conversations during the preparation of this paper. 


\section{Notation and definitions}

The open unit disk in the complex plane will be denoted by $\mathbb{Q}$ and its boundary, the unit circle, by $\partial \mathbb{Q}$. The set of analytic functions $f$ on $\mathbb{O}$ into $\mathbb{O}$ with the property that $\left|\lim _{r \rightarrow 1} f\left(r e^{i \theta}\right)\right|=1$ for Lebesgue almost all $\theta$ are the inner functions on the unit disk. Every inner function has a representation of the form

$$
f(z)=c\left(\prod_{j=1}^{\infty} \frac{\left|a_{j}\right|}{a_{j}} \frac{z-a_{j}}{1-\bar{a}_{j} z}\right) \exp \left\{-\int_{\partial \Theta} \frac{\zeta+z}{\zeta-z} d \nu(\zeta)\right\}
$$

where $|c|=1,\left|a_{j}\right|<1, \sum_{j=1}^{\infty}\left(1-\left|a_{j}\right|\right)<\infty$, and $\nu$ is a positive measure on 20 singular with respect to Lebesgue measure. In this formula, if $a_{j}=0,\left|a_{j}\right| / a_{j}$ is taken to be one (see [9]).

A Blaschke product is an inner function in which the singular measure $\nu$ in (1) is identically zero and a singular inner function is one with no zeros in $\mathbb{O}$, i.e., the Blaschke product in (1) is missing.

It is clear from the representation (1) that an inner function is not only analytic on $\mathbb{O}$ but also on the complement of $\mathbb{Q} \cup \partial \mathbb{Q}$ except at the poles $\left(\bar{a}_{j}\right)^{-1}, j=1,2, \ldots$ It is not defined at the accumulation points of the zeros $a_{j}$ and on the closed support of the measure $\nu$. These points are essential singularities of $f$. A general inner function may not be defined at any point of $2 \mathbb{Q}$; however, by Fatou's theorem $f^{*}\left(e^{i \theta}\right)=$ $\lim _{-r \rightarrow 1} f\left(r e^{i \theta}\right)$ exists for almost all $\theta$ and by assumption $\left|f^{*}\left(e^{i \theta}\right)\right|=1$ so that $f^{*}$ is

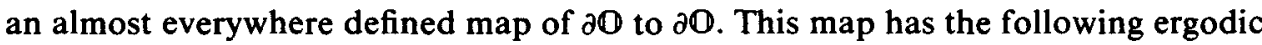
properties:

(1) If $f^{*}$ is nonconstant, it is nonsingular and positively nonsingular with respect to Lebesgue measure $\lambda$ on $\partial \mathbb{Q}$, i.e., $\lambda(A)=0$ implies that both $\lambda\left(f^{*-1}\{A\}\right)=0$ and $\lambda\left(f^{*}\{A\}\right)=0([8])$.

(2) If $f$ is not a Möbius transformation, there exists a unique fixed point $\delta$ in $\mathbb{Q} \cup \partial \mathbb{O}$ such that $\left|f^{\prime}(\delta)\right| \leq 1$ if $\delta \in \mathbb{O},\left|\lim _{r \rightarrow 1-} f^{\prime}(r \delta)\right| \leq 1$ if $\delta \in \partial \mathbb{O}$ and for $z \in \mathbb{O}$, $\lim _{n \rightarrow \infty} f^{n}(z)=\delta$, where $f^{n}$ is the composition of $f$ with itself $n$ times. The point $\delta$ is called the Denjoy-Wolff point for $f([21,4])$.

(3) If $f$ has a fixed point in $\mathbb{O}$ and $f$ is not a Möbius transformation, then it is the Denjoy-Wolff point $\delta$ (hence unique) and $\left|f^{\prime}(\delta)\right|<1$ (Schwartz Lemma; [9]).

(4) If the Denjoy-Wolff point $\delta$ is in $\mathbb{O}$ and $f$ is not Möbius, then $f^{*}$ has a unique finite invariant measure equivalent to Lebesgue measure whose density is the Poisson Kernel at $\delta$, i.e., $P_{\delta}(\zeta)=\left(1-|\delta|^{2}\right) /|\zeta-\delta|^{2}$ for $\zeta \in \partial \mathbb{Q}$, and $f^{*}$ is an exact endomorphism.

If $\delta \in \partial \mathbb{O}$, then $f^{*}$ does not have a finite invariant measure absolutely continuous with respect to Lebesgue measure, although it may have an equivalent $\sigma$-finite invariant measure. If $\left|\lim _{r \rightarrow 1-} f^{\prime}(r \delta)\right|<1, f^{*}$ is not ergodic. If $\left|\lim _{-r \rightarrow 1} f^{\prime}(r \delta)\right|=1, f^{*}$ can be either exact or not ergodic $([1,2,10,14,17])$.

In case $f$ is continuous on $\partial \mathbb{O}$, then $f^{*}$ is the restriction of $f$ to $\partial \mathbb{O}$ and $f$ is a finite Blaschke product, i.e.,

$$
f(z)=c \prod_{j=1}^{k} \frac{z-a_{j}}{1-\bar{a}_{j} z}, \quad\left|a_{j}\right|<1,|c|=1 .
$$

In this case $f^{*}$ is a $C^{\infty}$ map of the compact manifold $\partial \mathbb{O}$ to itself and its ergodic 
properties can be studied by using results from smooth dynamics. In particular, if $\left|f^{\prime}(\zeta)\right|>1$ for all $\zeta \in \partial \mathbb{O}$, then $f^{*}$ is an expanding map. This observation was developed in [11]. Also see [20].

In the following we restrict ourselves to the class $\mathscr{F}$ of inner functions which have a finite nonempty set of singularities on $\partial \mathbb{O}$. If $f \in \mathscr{F}$, the singularities of $f$ on $\partial \mathbb{O}$ will be denoted by $\mathbb{E}(f)$, or just $\mathbb{E}$. If $f \in \mathscr{F}, f^{*}=f$ on $\partial \mathbb{O}-\mathbb{E}(f)$ and $f$ cannot be extended continuously to any point in $\mathbb{E}(f)$.

Since $\mathbb{E}(F)$ is finite for $f \in \mathscr{F}$, we can represent the function in $\mathscr{F}$ by

$$
f(z)=c\left(\prod_{j=1}^{\infty} \frac{\left|a_{j}\right|}{a_{j}} \frac{z-a_{j}}{1-\bar{a}_{j} z}\right) \exp \left\{-\sum_{i=1}^{N} \frac{b_{l}+z}{b_{l}-z} m_{l}\right\},
$$

where $c, a_{j}$ are as in (1), $\left|b_{l}\right|=1, m_{l}$ are positive and the closure of the $a_{j}$ 's intersected with $\partial O$ is finite. For these maps $\log f$ has a well-defined analytic branch in some neighbourhood $U$ of $\zeta \in \partial \mathbb{O}-\mathbb{E}$ and

$$
(\log f)^{\prime}(z)=\sum_{j=1}^{\infty} \frac{1-\left|a_{j}\right|^{2}}{\left(z-a_{j}\right)\left(1-\bar{a}_{j} z\right)}+\sum_{l=1}^{N} \frac{-b_{l} m_{l}}{\left(b_{l}-z\right)^{2}}
$$

for $z \in U$.

If $(\Omega, \mathscr{B}, \mu)$ is a Lebesgue space and $T$ is a measure-preserving transformation on $\Omega$, a measurable partition $\eta$ of $\Omega$ is a one-sided generator if $\bigvee_{j=0}^{\infty} T^{-j} \eta=\varepsilon(\bmod 0)$, where $\varepsilon$ denotes the point partition and $\bigvee$ the common refinement. If $T$ is a countable to one endomorphism of $\Omega$, then for each $\omega \in \Omega, \varepsilon \cap T^{-1}\{\omega\}$ is a countable partition of the atom $T^{-1}\{\omega\}$ of $T^{-1} \varepsilon$. By Rohlin's theorem there exists a countable measurable partition $\gamma$ of $\Omega$ such that $\varepsilon=\varepsilon \vee T^{-1} \varepsilon=\gamma \bigvee T^{-1} \varepsilon(\bmod 0)$, and $T$ is injective on each atom of $\gamma$. The conditional information of $\varepsilon$ given $T^{-1} \varepsilon$ is defined by $I\left(\varepsilon \mid T^{-1} \varepsilon\right)=I\left(\gamma \mid T^{-1} \varepsilon\right)$. Since $T$ is injective on each atom in $\gamma, T_{A}$, the restriction of $T$ to $A \in \gamma$, defines a measure $\mu T_{A}$ which is nonsingular with respect to $\mu$. The Jacobian of $T$ is defined by

$$
J_{T}(\omega)=\sum_{A \in \gamma} \chi_{A}(\omega) \frac{d \mu T_{A}}{d \mu}(\omega) .
$$

A connection between $J_{T}$ and $I\left(\varepsilon \mid T^{-1} \varepsilon\right)$ is given by

$$
I\left(\varepsilon \mid T^{-1} \varepsilon\right)(\omega)=\log J_{T}(\omega) .
$$

In case the integral of $I\left(\varepsilon \mid T^{-1} \varepsilon\right)$ is defined, the conditional entropy is given by

$$
H\left(\varepsilon \mid T^{-1} \varepsilon\right)=\int_{\Omega} I\left(\varepsilon \mid T^{-1} \varepsilon\right) d \mu=\int_{\Omega} \log J_{T} d \mu .
$$

Moreover, the entropy of $T, h(T)$, satisfies

$$
h(T) \geq H\left(\varepsilon \mid T^{-1} \varepsilon\right)
$$

with equality if $T$ has a one-sided generator with finite entropy. If more than one measure is being considered, we will write $I_{\mu}\left(\varepsilon \mid T^{-1} \varepsilon\right)$ and $H_{\mu}\left(\varepsilon \mid T^{-1} \varepsilon\right)$ for the conditional information and entropy with respect to the measure $\mu$. For details of entropy, information, and the Jacobian one may consult [15] and [13].

In the following, $m$ will denote Lebesgue measure on $[0,1]$ and $\lambda$ will denote normalized Lebesgue measure on $\partial \mathbb{O}$. If $f$ is a map of a set $X$ to itself, then $f^{n}(x)$ 
will denote the composition of $f$ with itself $n$ times, i.e., $f^{0}(x)=x, f^{n}(x)=f \circ f^{n-1}(x)$ for $n>1$. If $f$ is differentiable at $x, f^{\prime}(x)$ denotes the first derivative and $f^{(n)}(x)$ denotes the $n$th derivative for $n>1$.

If $f$ is a complex-valued function on an interval of real numbers, $f$ is of bounded variation if

$$
\sup \left\{\sum_{i=1}^{k}\left|f\left(x_{i}\right)-f\left(x_{i-1}\right)\right|\right\}<\infty
$$

where the sup is taken over all partitions $x_{0}<x_{1}<\cdots<x_{k}$ of the interval. The Nevalinna class $N$ is the collection of all analytic functions $h$ on $\mathbb{O}$ such that

$$
\sup _{0<r<1}\left\{\int_{-\pi}^{\pi} \log ^{+}\left|h\left(r e^{\mathrm{i} \theta}\right)\right| \frac{\mathrm{d} \theta}{2 \pi}\right\} \leq B
$$

\section{Inner functions whose natural extensions are Bernoulli}

LEMMA 2.1. If $f \in \mathscr{F}$, then

(1) there exists an open dense set $U$ in $[0,1]$ such that $m(U)=1$;

(2) there exists a countable family $\beta$ of closed intervals in $[0,1]$ with disjoint interiors such that $\bigcup\{B: B \in \beta\} \supset U$ and for any $B \in \beta$ the set $B \cap\{[0,1]-U\}$ consists exactly of the end points of $B$;

(3) there exists a differentiable map $T$ of $U$ to $[0,1]$ such that for any $B \in \beta, T$ restricted to $U \cap B$ admits an extension to a diffeomorphism of $B$ into $[0,1]$ and

(4) for every $B \in \beta, t \in B$

$$
f(\exp 2 \pi \mathrm{i} t)=\exp 2 \pi \mathrm{i} T(t)
$$

and

$$
\left|f^{\prime}(\exp 2 \pi \mathrm{i} t)\right|=T^{\prime}(t) .
$$

Proof. Let $f \in \mathscr{F}$ and $\mathbb{E}(f)=\left\{\exp 2 \pi i c_{j}: j=0,1, \ldots, K-1\right\}$ with $c_{0}=0$. Define

$$
C_{j}=\left\{\exp (2 \pi \mathrm{i} t): c_{j}<t<c_{j+1}\right\}
$$

for $j=0,1, \ldots, K-1$ with $c_{K}=1$. Denote the mod-point of $C_{j}$ by $\exp 2 \pi \mathrm{i} t_{j}$.

For each $j, f$ is nonzero and analytic on a simply connected open set $V_{j}$ containing $C_{j}$, and there exists a well-defined analytic branch of $\log f$ on $V_{j}$, say

$$
\log f_{j}(z)=\psi_{j}(z)+\mathrm{i} \alpha_{j}(z) \quad z \in V_{j}
$$

with $\alpha_{j}\left(\exp 2 \pi \mathrm{i} t_{j}\right)=0$ and $\psi_{j}(z)=0$ on $V_{j} \cap \partial \mathbb{O}$.

If $f$ has the representation (2), then

$$
\left(\log f_{j}\right)^{\prime}\left(e^{2 \pi \mathrm{i} t}\right)=e^{-2 \pi \mathrm{i} t}\left\{\sum_{k=1}^{\infty} \frac{1-\left|a_{k}\right|^{2}}{\left|e^{2 \pi \mathrm{i} t}-a_{k}\right|^{2}}+\frac{1}{2} \sum_{t=1}^{N} m_{l} \csc ^{2} \pi\left(\beta_{l}-t\right)\right\},
$$

where $\exp 2 \pi i \beta_{l}=b_{l}, \beta_{l} \in[0,1]$. Since $|f|=1$ on $\partial \mathbb{O}-\mathbb{E}$,

$$
\left|f^{\prime}\left(e^{2 \pi \mathrm{i} t}\right)\right|=\sum_{k=1}^{\infty} \frac{1-\left|a_{k}\right|^{2}}{\left|e^{2 \pi \mathrm{i} t}-a_{k}\right|^{2}}+\frac{1}{2} \sum_{t=1}^{N} m_{l} \csc ^{2} \pi\left(\beta_{l}-t\right)
$$

and

$$
\left(\log f_{j}\right)^{\prime}\left(e^{2 \pi \mathrm{i} t}\right)=e^{-2 \pi \mathrm{i} t}\left|f^{\prime}\left(e^{2 \pi \mathrm{i} t}\right)\right|
$$


For $t \in\left(c_{j}, c_{j+1}\right)$, define

$$
A_{j}(t)=\frac{1}{2 \pi} \alpha_{j}\left(e^{2 \pi \mathrm{i} t}\right)
$$

Then

$$
\begin{aligned}
A_{j}^{\prime}(t) & =\frac{d}{d t}\left[\frac{1}{2 \pi i} \log f_{j}\left(e^{2 \pi \mathrm{i} t}\right)\right] \\
& =e^{2 \pi \mathrm{i} t}\left(\log f_{j}\right)^{\prime}\left(e^{2 \pi \mathrm{i} t}\right) \\
& =\left|f^{\prime}\left(e^{2 \pi \mathrm{i} t}\right)\right|,
\end{aligned}
$$

and $A_{j}$ is differentiable and strictly increasing on $\left(c_{j}, c_{j+1}\right)$ with $A_{j}\left(t_{j}\right)=0$.

Let $I_{j, k}=\left\{t \in\left(c_{j}, c_{j+1}\right): k \leq A_{j}(t) \leq k+1\right\}$ for $j=0,1,2, \ldots, K-1$ and $k=$ $0, \pm 1, \pm 2, \ldots$ Define $T$ on $\cup I_{j, k}^{0}$ by

$$
T(t)=A_{j}(t)-k \text { for } t \in I_{j, k} \text {. }
$$

Take $\beta=\left\{I_{j, k}: 0 \leq j<K, k \in \mathbb{Z}\right\}, U=\bigcup I_{j, k}^{0}$. Then for any $t \in I_{j, k}^{0}$

$$
T^{\prime}(t)=A_{j}^{\prime}(t)=\left|f^{\prime}(\exp 2 \pi \mathrm{i} t)\right|
$$

and

$$
\exp 2 \pi \mathrm{i} T(t)=\exp 2 \pi \mathrm{i}\left\{A_{j}(t)-k\right\}=f(\exp 2 \pi \mathrm{i} t) .
$$

Remark. A theorem of Lindelöf ([5] page 42) implies that for $f \in \mathscr{F}$, and $z \in \mathbb{E}(f)$, the limit of $f$ restricted to $\partial \mathbb{O}-\mathbb{E}(f)$ fails to exist from at least one side at $z$. Thus, since $A_{j}$ increases either $\lim _{t \rightarrow c_{j}+} A_{j}(t)=-\infty$ or $\lim _{t \rightarrow c_{j}-} A_{j}(t)=+\infty$. It follows from the definition of $T$ that for given $j, T$ maps $I_{j, k}$ onto $[0,1]$, either for all positive $k$ or for all negative $k$. If $\lim _{t \rightarrow c_{j}+} A_{j}(t)=-b_{j}$, then $T$ maps $I_{j, k}$ onto $\left[1-R_{j}+b_{j}, 1\right]$. The intervals $I_{j, k}$ for $k<-R_{j}$ are empty. A similar analysis can be made for the case where $\lim _{t \rightarrow c_{j+1}-} A_{j}(t)=b_{j}$. Thus $T$ maps all but at most $K$ intervals in $\beta$ onto $[0,1]$ where $K$ is the number of points in $\mathbb{E}(f)$.

Let

$$
C_{j, k}=\left\{\exp 2 \pi \mathrm{i} t: t \in I_{j, k}\right\}
$$

and $\eta$ denote the partition of $\partial \mathbb{O}$ given by the arcs $C_{j, k}$. thus for $f \in \mathscr{F}, f$ restricted to $C_{j, k}$ is a bijection to 20 .

Theorem 2.2. Let $f \in \mathscr{F}, \mathbb{E}(f)=\left\{\exp 2 \pi \mathrm{i} c_{j}: j=0,1, \ldots, K-1\right\}$. Assume

(i) there exists $N \geq 1$ such that

$$
\inf \left\{\left|\left(f^{N}\right)^{\prime}(z)\right|: z \in C\right\}>1
$$

for each $C \in \eta^{N}=\bigvee_{j=0}^{N-1} f^{-j} \eta$

(ii) $\left[f^{\prime}\left(e^{2 \pi \mathrm{i} t}\right)\right]^{-1}$ is of bounded variation on $\left(c_{j}, c_{j+1}\right)$ for $j=0,1,2, \ldots, K-1$.

Then the Denjoy-Wolff point $\delta$ for $f$ is in 0 so that $d \mu=P_{\delta}(\zeta) d \lambda(\zeta)$ is $f$-invariant and the system $(\partial \mathrm{O}, f, \mu)$ is exact and its natural extension is Bernoulli.

Proof. Let $T, U, \beta$ denote respectively the map, open dense set of $[0,1]$, and partition of $[0,1]$ associated with $f$ by Lemma 2.1. Define $g(t)$ on $[0,1]$ by

$$
g(t)= \begin{cases}\left|f^{\prime}\left(e^{2 \pi \mathrm{i} t}\right)\right|^{-1} & \text { if } t \in I_{j, k}^{0} \in \beta . \\ 0 & \text { otherwise. }\end{cases}
$$


Since $\left[f^{\prime}(\exp 2 \pi i t)\right]^{-1}$ is of bounded variation on $\left(c_{j}, c_{j+1}\right), g(t)$ is of bounded variation on $[0,1]$.

$$
\begin{aligned}
& \text { If } g_{N}(t)=\prod_{j=0}^{N-1} g\left(T^{j}(t)\right) \text {, then } g_{N}(t)=\left|\left(f^{N}\right)^{\prime}\left(e^{2 \pi \mathrm{i} t}\right)\right|^{-1} \text { a.e. so that }\left\|g_{N}\right\|_{\infty}<1 \text {. Let } \\
& U^{N}=[0,1]-\bigcup_{j=1}^{N} T^{-j}([0,1]-U), \quad \beta^{N}=\bigvee_{j=0}^{N-1} T^{-j} \beta
\end{aligned}
$$

Then $U^{N}$ is an open dense set in $[0,1]$ such that $m\left(U^{N}\right)=1, \beta^{N}$ is a countable family of closed intervals in $[0,1]$ with disjoint interiors such that $\bigcup\left\{B: B \in \beta^{N}\right\} \supset$ $U^{N}$ and for any $B \in \beta^{N}$ the set $B \cap\left\{[0,1]-U^{N}\right\}$ consists exactly of the end points of $B ; T^{N}$ is a differentiable map of $U^{N}$ to $[0,1]$ such that for any $B \in \beta^{N}, T^{N}$ restricted to $U^{N} \cap B$ admits an extension to a diffeomorphism of $B$ into $[0,1]$. Corollary 1 of [19], together with remarks preceding this corollary imply that $T^{N}$, $U^{N}, g_{N}$ and $\beta^{N}$ satisfy the hypothesis on the maps considered in [19]. Let $\tau_{B}$ denote $T^{N}$ restricted to $B$ for $B \in \beta^{N}$. Then for $x \in T^{N} B$.

$$
\left|\left(\tau_{B}^{-1}\right)^{\prime}(x)\right|=\left|\left(T^{N}\right)^{\prime}\left(\tau_{B}^{-1}(x)\right)\right|^{-1} .
$$

For $\varphi \in L_{1}(d m)$, let

$$
P \varphi(x)=\sum_{y \in T x} g_{N}(t) \varphi(y) .
$$

Since $g_{N}(y)=\left|\left(T^{N}\right)^{\prime}(y)\right|^{-1}$, we can write

$$
\begin{aligned}
P \varphi(x) & =\sum_{B \in \beta^{N}} X T^{-N}\{x\} \cap B(y) \varphi(y)\left|\left(T^{N}\right)^{\prime}(y)\right|^{-1} \\
& =\sum_{B \in \beta^{N}} \chi_{B}(y) \chi_{\{x\}}\left(T^{N} y\right) \varphi(y)\left|\left(T^{N}\right)^{\prime}(y)\right|^{-1} \\
& =\sum_{B \in \beta^{N}} \chi_{T^{N} B}(x) \varphi\left(\tau_{B}^{-1}(x)\right)\left|\left(T^{N}\right)^{\prime}\left(\tau_{\beta}^{-1}(x)\right)\right|^{-1} .
\end{aligned}
$$

Since $\beta^{N}$ is a countable partition

$$
\begin{aligned}
\int P \varphi(x) d m(x) & =\sum_{B \in \beta^{N}} \int_{\tau_{B} B} \varphi\left(\tau_{B}^{-1}(x)\right)\left|\left(T^{N}\right)^{\prime}\left(\tau_{B}^{-1}(x)\right)\right|^{-1} d m(x) \\
& =\int \varphi(y) d m(y) .
\end{aligned}
$$

It now follows as in Remark 2 of [19] that for $\varphi, \psi$ in $L_{1}(d m), P\left(\varphi\left(T^{N}\right) h\right)=\varphi P(h)$.

Theorem 1 of [19] proves among other things that if $P$ is considered as an operator on BV (the set of $L_{\infty}$ functions with a version of bounded variation) then 1 is an eigenvalue for $P$ so there exists a non-zero $L_{\infty}$ function $h$ such that $P h=h$. Let $d \tilde{\mu}=h d m$. For any $\varphi \in C([0,1])$

$$
\begin{aligned}
\int\left(\varphi \cdot T^{N}\right) h d m & =\int P\left(\left(\varphi \cdot T^{N}\right) h\right) d m \\
& =\int \varphi P h d m \\
& =\int \varphi h d m
\end{aligned}
$$


i.e.,

$$
\int\left(\varphi \cdot T^{N}\right) d \tilde{\mu}=\int \varphi d \tilde{\mu}
$$

and $\tilde{\mu}$ is a $T^{N}$ invariant measure absolutely continuous with respect to $m$. Since $\exp 2 \pi \mathrm{i} T^{N}(t)=f^{N}(\exp 2 \pi \mathrm{i} t), f^{N}$ has an absolutely continuous invariant probability $\mu$ on $\partial \mathbb{O}$. Since $f^{N}$ is an inner function (5) implies that $d \mu=P_{\delta_{N}} d \lambda$ where $\delta_{N} \in \mathbb{O}$ is the Denjoy-Wolff point for $f^{N}$, and $f^{N}$ is an exact endomorphism of $\partial \Theta$, and hence $T^{N}$ is an exact endomorphism of $[0,1]$. Article 3 of [19] shows that since $T^{N}$ is exact, it has a Bernoulli natural extension, so $f^{N}$ has a Bernoulli natural extension with respect to $\mu$. Let $\delta$ denote the Denjoy-Wolff point for $f$. Since $f^{N}\left(\delta_{N}\right)=\delta_{N}$ and $\lim _{n \rightarrow \infty} f^{n}\left(\delta_{N}\right)=\delta, \delta=\delta_{N}$ and $\mu$ is an absolutely continuous invariant measure for $f$ on $\partial \mathbb{O}$. If $\tau$ denotes the natural extension of $\left(f^{N}, \mu\right), \tau^{n}$ is the natural extension of $\left(f^{N}, \mu\right)$, and since roots of Bernoulli automorphisms are Bernoulli, $\tau$ is Bernoulli.

LEMMA 2.3. If $h$ is analytic on a smooth path $\gamma:(a, b) \rightarrow \mathbb{C}$, and $\left(h^{\prime} \circ \gamma\right) \gamma^{\prime} \in L^{\mathbf{1}}(m)$, then $h \circ \gamma$ is of bounded variation on $(a, b)$.

Proof. Define a complex measure $\mu$ on $[a, b]$ by

$$
\mu(A)=\int_{A} h^{\prime}(\gamma(t)) \gamma^{\prime}(t) d m(t) .
$$

The total variation measure $|\mu|$ of $\mu$ (see Rudin [18] page 116) satisfies

$$
|\mu|(E)=\sup \left\{\sum_{j=1}^{\infty}\left|\mu\left(E_{j}\right)\right|\right\}
$$

where the sup is taken over all partitions $\left\{E_{j}: j=1,2, \ldots\right\}$ of the set $E$, and $|\mu|$ $[a, b]<\infty$.

Let $a<x_{0}<x_{1}<\cdots<x_{n}<b$ be given. Then

$$
\begin{aligned}
\sum_{j=1}^{n}\left|h\left(\gamma\left(x_{j}\right)\right)-h\left(\gamma\left(x_{j-1}\right)\right)\right| & =\sum_{j=1}^{n}\left|\int_{x_{j-1}}^{x_{j}} h^{\prime}(\gamma(t)) \gamma^{\prime}(t) d m(t)\right| \\
& =\sum_{j=1}^{n}\left|\mu\left(x_{j}, x_{j-1}\right)\right| \\
& \leq|\mu|(a, b)
\end{aligned}
$$

and $h \circ \gamma$ is of bounded variation on $(a, b)$.

Remark 2.4. Suppose $f$ is a singular inner function in $\mathscr{F}$, say

$$
f(z)=\exp \left\{\sum_{l=0}^{K-1} m_{l} \frac{e^{2 \pi i c_{l}}+z}{e^{2 \pi i c_{l}}-z}\right\}
$$

for $0 \leq c_{l} \leq 1$. Then, if $h(t)=\left|f^{\prime}(\exp 2 \pi \mathrm{i} t)\right|^{-1}$,

Let

$$
h^{\prime}(t)=\frac{4 \sum_{l=1}^{K-1} m_{l} \csc ^{2} \pi\left(c_{l}-t\right) \cot \pi\left(c_{l}-t\right)}{\left[\sum_{l=0}^{K-1} m_{l} \csc ^{2} \pi\left(c_{l}-t\right)\right]^{2}}
$$

$$
h_{j}(t)=\frac{4 m_{j} \csc ^{2} \pi\left(c_{j}-t\right) \cot \pi\left(c_{j}-t\right)}{\left[m_{j} \csc ^{2} \pi\left(c_{j}-t\right)\right]^{2}}, j=0,1, \ldots, K-1 .
$$


then

$$
\lim _{t \rightarrow c_{j}} \frac{h^{\prime}(t)}{h_{j}(t)}=1
$$

and since $\lim _{t \rightarrow c_{j}} h_{j}(t)=0, \lim _{t \rightarrow c_{j}} h^{\prime}(t)=0$. Since $h^{\prime}$ is continuous on $\left(c_{j}, c_{j+1}\right)$, it is bounded and it follows from Lemma 2.3 that $\left[f^{\prime}(\exp 2 \pi \mathrm{i} t)\right]^{-1}$ is of bounded variation. Thus hypothesis (ii) of Theorem 2.2 is satisfied for singular inner functions.

Notice also that if $f \in \mathscr{F}$ and $f^{\prime \prime}(z)\left[f^{\prime}(z)\right]^{-2}$ is in $L_{1}(\partial \mathbb{O}, \lambda)$ then hypothesis (ii) of Theorem 2.2 is also satisfied. This is true because if $\gamma(t)=\exp (2 \pi \mathrm{i} t)$ and $h(z)=$ $\left[f^{\prime}(z)\right]^{-1}$, then

$$
h^{\prime}(\gamma(t)) \gamma^{\prime}(t)=2 \pi \mathrm{i} f^{\prime \prime}\left(e^{2 \pi \mathrm{i} t}\right)\left[f^{\prime}\left(e^{2 \pi \mathrm{i} t}\right)\right]^{-2} e^{2 \pi \mathrm{i} t}
$$

is in $L_{1}(m)$ and the claim follows from Lemma 2.3 .

\section{Entropy of inner functions}

If $f \in \mathscr{F}$, Lemma 2.1 associates a partition $\eta$ of $\partial \mathbb{O}$ into arcs $C$ such that $f$ is a diffeomorphism of $C$ to $\partial \mathbb{Q}$.

\section{THEOREM 3.1. Let $f \in \mathscr{F}$ and assume}

(i) the Denjoy-Wolff point $\delta$ for $f$ is in $\mathbb{O}$,

(ii) $\inf \left\{\left|f^{\prime}(z)\right|: z \in C\right\} \lambda(C) \geq P>0$ for all atoms $C \in \eta$.

Then the entropy of $f$ with respect to the measure $P_{\delta} d \lambda$ is given by

$$
h(f)=\int_{\partial \Phi} P_{\delta}(\zeta) \log \left|f^{\prime}(\zeta)\right| d \lambda(\zeta) .
$$

Proof. Since $\delta \in \mathbb{O}, d \mu=P_{\delta} d \lambda$ is invariant and $(\partial \mathbb{O}, f, \mu)$ is exact. Thus, for any nontrivial partition $\xi$, the $\sigma$-algebra of the partition $\bigvee_{k=0}^{\infty} f^{-k} \xi$ is nonatomic and in particular $\lim _{n \rightarrow \infty} \mu\left(\cap_{k=0}^{n} f^{-k} C_{k}\right)=0$ for any selection of $C_{k}$ in $\eta$. Since $\mu$ is equivalent to $\lambda$ it follows that $\lim _{n \rightarrow \infty} \lambda\left(\bigcap_{k=0}^{n} f^{-k} C_{k}\right)=0$.

The atom

$$
C_{0} \cap f^{-1} C_{1} \cap \cdots \cap f^{-n} C_{n}=C_{0} \cap f_{0}^{-1} C_{1} \cap \cdots \cap f_{0}^{-n} C_{n},
$$

where $f_{0}=f \mid C_{0}$, and since $f_{0}$ is a homeomorphism, this intersection is an arc in $\partial \mathbb{O}-\mathbb{E}(f)$, and the diameters of these arcs go to zero as $n \rightarrow \infty$. Thus, if $x, x^{\prime}$ are in the same atom of $\bigvee_{k=0}^{\infty} f^{-k} \eta, x=x^{\prime}$, and $\eta$ is a one-sided generator for $f$.

Let $\eta=\left\{C_{k}: k=0,1,2, \ldots\right\}$. Since $\sum \lambda\left(C_{k}\right)=1, \lim _{k \rightarrow 0} \lambda\left(C_{k}\right)=0$ and there is $K>0$ such that for all $k \geq K, \lambda\left(C_{k}\right)<P$. By hypothesis (ii), $\left|f^{\prime}(z)\right| \geq P / \lambda\left(C_{k}\right)$ for all $z \in C_{k}$ so that

$$
\log \left|f^{\prime}(z)\right| \geq \log \frac{P}{\lambda\left(C_{k}\right)}>0
$$

for all $z \in C_{k}, k \geq K$. Thus $\left|f^{\prime}(z)\right|$ is bounded from below and the integral of $\log \left|f^{\prime}(z)\right|$ exists. Moreover,

$$
\int_{C_{k}} \log \left|f^{\prime}\right| d \lambda \geq \lambda\left(C_{k}\right) \log \frac{P}{\lambda\left(C_{k}\right)}
$$


for all $k$, so that

$$
\int_{\partial \Phi} \log \left|f^{\prime}\right| d \lambda \geq H_{\lambda}(\eta)+\log P
$$

Now if $f_{k}$ denotes the restriction of $f$ to an atom $C_{k}$ of $\eta, h$ is continuous on 20 and $\boldsymbol{A}$ is a Borel set in $\partial \mathbb{O}$. Then

$$
\begin{aligned}
\int_{f_{k} A} h(t) d \mu(t) & =\int_{f_{k} A} h(t) P_{\delta}(t) d \lambda(t) \\
& =\int_{A}\left(h \cdot f_{k}\right)\left(P_{\delta} \cdot f_{k}\right)\left|f_{k}^{\prime}\right| d \lambda \\
& =\int_{A}\left(h \cdot f_{k}\right)\left(P_{\delta} \cdot f_{k}\right)\left(P_{\delta}\right)^{-1}\left|f_{k}^{\prime}\right| d \mu .
\end{aligned}
$$

Thus the Jacobian $J_{\mu}$ is given by

$$
J_{\mu}(z)=\frac{P_{\delta}(f(z))}{P_{\delta}(z)}\left|f^{\prime}(z)\right| \quad \text { a.e. }
$$

Since $\int_{\partial \mathbf{O}} \log \left|f^{\prime}\right| d \lambda$ exists and $\mu$ has positive density with respect to $\lambda, \int_{\partial \mathrm{O}} \log \left|f^{\prime}\right| d \mu$ exists and

$$
\begin{aligned}
H_{\mu}\left(\varepsilon \mid f^{-1} \varepsilon\right) & =\int_{\partial \mathbf{0}} \log J_{\mu}(z) d \mu \\
& =\int_{\partial \mathbf{\Phi}} \log \left(P_{\sigma} \cdot f\right) d \mu-\int_{\partial \mathbf{0}} \log P_{\sigma} d \mu+\int_{\partial \mathbf{0}} \log \left|f^{\prime}\right| d \mu \\
& =\int_{\partial \mathbf{0}} \log \left|f^{\prime}\right| d \mu
\end{aligned}
$$

since $\mu$ is $f$-invariant.

If $\log \left|f^{\prime}\right|$ is integrable on $\partial \mathbb{O}$, then by (4) $H_{\lambda}(\eta)<\infty$ and since $\mu$ has a bounded density function with respect to $\lambda, H_{\mu}(\eta)<\infty$. Thus $\eta$ is a one-sided generator with finite $\mu$-entropy and

$$
h_{\mu}(f)=H_{\mu}\left(\varepsilon \mid f^{-1} \varepsilon\right)=\int_{\partial \circlearrowright} P_{\delta}(z) \log \left|f^{\prime}(z)\right| d \lambda(z) .
$$

If $\log \left|f^{\prime}\right|$ is not integrable, since $H_{\lambda}(\eta) \geq 0$, (4) implies that $\int_{\partial \mathbf{O}} \log \left|f^{\prime}\right| d \mu=+\infty$ and

$$
h_{\mu}(f) \geq H_{\mu}\left(\varepsilon \mid f^{-1} \varepsilon\right)=\int_{\partial \Theta} \log \left|f^{\prime}\right| d \mu=+\infty .
$$

LEMMA 3.2. If $f \in \mathscr{F}$ is such that

$$
\sup \left\{\left|f^{\prime}\left(z_{1}\right)\right| /\left|f^{\prime}\left(z_{2}\right)\right|: z_{1}, z_{2} \in C\right\} \leq Q
$$

for every $C \in \eta$, then there exists $P>0$ such that

$$
\inf \left\{\left|f^{\prime}(z)\right|: z \in C\right\} \lambda(C) \geq P \text {. }
$$

Proof. Let $C \in \eta$. Since $f$ restricted to $C$ is a diffeomorphism

$$
\int_{C}\left|f^{\prime}\right| d \lambda=\lambda(f(C))
$$


By the mean value theorem for integrals there exists $z_{1} \in C$ such that

$$
\left|f^{\prime}\left(z_{1}\right)\right| \lambda(C)=\lambda(f(C)) \text {. }
$$

Since $\lambda(f(C))>0$ and is equal to one for all but a finite number of $C \in \eta$, there exists $\tilde{P}>0$ such that

$$
\left|f^{\prime}\left(z_{1}\right)\right| \lambda(C)>\tilde{P} \text { for all } C \in \eta
$$

Let $P=\tilde{P} Q^{-1}$. For any $z \in C$

$$
\begin{aligned}
\left|f^{\prime}(z)\right| \lambda(C) & =\left|f^{\prime}\left(z_{1}\right)\right| \lambda(C) \frac{\left|f^{\prime}(z)\right|}{\left|f^{\prime}\left(z_{1}\right)\right|} \\
& \geq \tilde{P}\left(\inf \left\{\frac{\left|f^{\prime}\left(z_{2}\right)\right|}{\left|f^{\prime}\left(z_{1}\right)\right|}: z_{1}, z_{2} \in C\right\}\right) \\
& \geq P .
\end{aligned}
$$

COROLlary 3.3. Let $f \in \mathscr{F}$ be such that

(i) there exists $N \geq 1$ such that

$$
\inf \left\{\left|\left(f^{N}\right)^{\prime}(z)\right|: z \in C\right\}>1 \quad \text { all } C \in \eta
$$

(ii) $f^{\prime \prime} /\left(f^{\prime}\right)^{2} \in L^{1}(\partial \mathbb{O}, \lambda)$,

and

(iii) $\sup \left\{\left|f^{\prime}\left(z_{1}\right)\right| /\left|f^{\prime}\left(z_{2}\right)\right|: z_{1}, z_{2} \in C\right\} \leq Q$ all $C \in \eta$.

Then

(1) there exists an invariant probability measure $\mu$ on $\partial \mathbb{O}$ given by $d \mu=P_{\delta} d \lambda$ where $\delta \in \mathbb{O}$ and $f(\delta)=\delta$,

(2) $(\partial \mathrm{O}, f, \mu)$ is exact and has a Bernoulli natural extension,

(3) $h_{\mu}(f)=\int_{\partial \Theta} P_{\delta}(z) \log \left|f^{\prime}(z)\right| d \lambda(z)$.

Proof. Follows from Remark 2.4, Lemma 3.2, and Theorem 3.1.

LEMMA 3.4. If $h$ is in the Nevalinna class $N, z_{0} \in \mathbb{O}$, and $h$ has a zero of order $d$ at $z_{0}$ then

$$
\begin{aligned}
\int_{\partial \mathrm{O}} P_{z_{0}}(\zeta) \log |h(\zeta)| d \lambda(\zeta)= & \log \frac{\left(1-\left|z_{0}\right|^{2}\right)^{d}}{d !}\left|h^{(d)}\left(z_{0}\right)\right| \\
& +\sum_{j=1}^{\infty} \log \left|\frac{1-b_{j} \bar{z}_{0}}{z_{0}-b_{j}}\right|-\int_{\partial \mathrm{Q}} P_{z_{0}}(\zeta) d \mu_{s}(\zeta)
\end{aligned}
$$

where $\left\{b_{j}: j=1,2, \ldots\right\}$ are the zeros of $h$ in $\mathbb{O}$ which are different from $z_{0}$, and $\mu_{s}$ is a signed measure on 20 singular with respect to Lebesgue measure on 20 .

Proof. Since $h$ is in the Nevanlinna class $N$, if $B$ denotes the Blaschke product formed from the zeros of $h$ in $\mathbb{O}$, and $g(z)=h(z) / B(z)$, then

$$
\log |g(z)|=\int P_{z}(t) d \mu(t)
$$

where $d \mu=\log |h| d \lambda+d \mu_{s}$. (See [9] page 70.)

Since $h$ has a zero of order $d$ at $z_{0} \in \mathbb{O}$,

$$
\frac{h(z)}{\left(z-z_{0}\right)^{d}}=\frac{h^{(d)}\left(z_{0}\right)}{d !}+0\left(z-z_{0}\right)
$$


and if the other zeros of $h$ are denoted by $\left\{b_{j}\right\}, j=1,2, \ldots$

$$
g(z)=h(z) \cdot\left(\frac{1-\bar{z}_{0} z}{z-z_{0}}\right)^{d} \prod_{j=1}^{\infty} \frac{1-\bar{b}_{j} z}{z-b_{j}}
$$

and

Thus

$$
g\left(z_{0}\right)=\frac{h^{(d)}\left(z_{0}\right)}{d !}\left(1-\left|z_{0}\right|^{2}\right)^{d} \prod_{j=1}^{\infty} \frac{1-\bar{b}_{j} z_{0}}{z_{0}-b_{j}} .
$$

$$
\begin{aligned}
\log \left|h^{(d)}\left(z_{0}\right) \frac{\left(1-\left|z_{0}\right|^{2}\right)^{d}}{d !}\right|+\sum_{j=1}^{\infty} \log \left|\frac{1-\bar{b}_{j} z_{0}}{z_{0}-b_{j}}\right| \\
=\int_{\partial \mathbb{Q}} P_{z_{0}}(\theta) \log |h(\theta)| d \lambda(\theta)+\int_{\partial \mathbb{Q}} P_{z_{0}}(\theta) d \mu_{s}(\theta)
\end{aligned}
$$

and

$$
\begin{aligned}
\int_{\partial \Theta} P_{z_{0}}(\theta) \log |h(\theta)| d \lambda(\theta)= & \log \left|\frac{h^{(d)}\left(z_{0}\right)\left(1-\left|z_{0}\right|^{2}\right)^{d}}{d !}\right| \\
& +\sum_{j=1}^{\infty} \log \left|\frac{1-\overline{b_{j}} z_{0}}{z_{0}-b_{j}}\right|-\int_{\partial Ф} P_{z_{0}}(\theta) d \mu_{s}(\theta) .
\end{aligned}
$$

\section{Corollary 3.5. Let $f \in \mathscr{F}$. Assume}

(i) the Denjoy-Wolff point $\delta$ for $f$ is in $\mathbb{O}$,

(ii) $f^{\prime} \in N$,

(iii) inf $\left\{\left|f^{\prime}(z)\right|: z \in C\right\} \lambda(C) \geq P>0$ for all atoms $C$ in $\eta$.

Then the entropy of $f$ with respect to the measure $d \mu=P_{\delta} d \lambda$ is given by

$$
h(f)=\log \left(\left|f^{(d)}(\delta)\right| \frac{\left(1-|\delta|^{2}\right)^{d-1}}{(d-1) !}\right)+\sum_{j=1}^{\infty} \log \left|\frac{1-\delta \bar{b}_{j}}{\delta-b_{j}}\right|-\int_{\partial \Theta} P_{\delta} d \nu,
$$

where $\nu$ is a signed measure singular with respect to Lebesgue measure on $\partial \mathbb{0},\left\{h_{j}\right\}$ are the zeros of $f^{\prime}$ in $\mathbb{O}$ different from $\delta$, and $d-1$ is the order of the zero of $f^{\prime}$ at $\delta$. [If $d=1, f^{\prime}(\delta) \neq 0$ ].

Remark. Ahern and Clark [3] show that if the zeros $\left\{a_{n}\right\}$ of a Blaschke product $f$ satisfy $\sum_{n=1}^{\infty}\left(1-\left|a_{n}\right|\right)^{r}<\infty$ for some $0<r<\frac{1}{2}$ then $f^{\prime} \in H^{1-r}$ and it follows that $f^{\prime} \in N$. Cullen [6] shows that if $f$ is a singular inner function in $\mathscr{F}$ then $f^{\prime} \in H^{p}$ for $0<p<\frac{1}{2}$ and hence $f^{\prime} \in N$. In either case if $f$ satisfies conditions (i) and (iii) of Corollary 3.5 , the entropy of $f$ is given by the formula in this corollary. Moreover the singular measure $\nu$ is a positive measure since in either case $f^{\prime} \in H^{P}$ for some $p>0$.

COROLLARY 3.6. If $f$ is a finite Blaschke product whose Denjoy-Wolff point is in $\mathbb{0}$ and $f^{\prime}(\delta) \neq 0$, then the entropy of $f \mid \partial \mathbb{O}$ is given by

$$
h(f)=\log \left|f^{\prime}(\delta)\right|-\sum_{j=1}^{K} \log \rho\left(\delta, b_{j}\right)
$$

where $\rho$ denotes the pseudo-hyperbolic metric in $\mathbb{O}$, and $b_{j}$ are the critical points of $f$ in $\mathbf{O}$ counted with their multiplicity.

Proof. Since $f$ is a finite Blaschke product, $f^{\prime}$ is non-zero and continuous on $\partial \mathrm{O}$, so that $f^{\prime}$ is bounded away from zero on $\partial \mathrm{O}$. Moreover the singular measure $\nu$ in 
Corollary 3.5 is zero. Since $\rho\left(\delta, b_{\delta}\right)=\left|\delta-b_{j}\right| /\left|1-\delta \bar{b}_{j}\right|$, and the critical points of $f$ in $\mathbb{O}$ are the zeros of $f^{\prime}$ in $\mathbb{O}$, the result is obtained from Corollary 3.5 .

An Example. Let

$$
f(z)=\exp c\left(\frac{z+1}{z-1}\right) \quad \text { for } c>0 .
$$

There is one singularity at $z=1$ so $\mathbb{E}=\{1\}$. There is a unique solution $\delta$ to $z=f(z)$ in $\mathbb{O}$, which is real, and this solution is the Denjoy-Wolff point for $f$.

Since

$$
f(\exp 2 \pi \mathrm{i} t)=\exp 2 \pi \mathrm{i}\left\{-\frac{c}{2 \pi} \cot \pi t\right\} \quad \text { for } 0<t<1
$$

the map on $[0,1]$ associated with $f$ by Lemma 2.1 is

$$
T(t)=-\frac{c}{2 \pi} \cot \pi t(\bmod 1) \quad 0<t<1
$$

and

$$
T^{\prime}(t)=\frac{c}{2} \csc ^{2} \pi t
$$

The intervals $I_{n}$ are

$$
\begin{aligned}
I_{n} & =\{t \in(0,1): n<T(t)<n+1\} \\
& =\left\{t: \frac{1}{\pi} \operatorname{arccot}\left(-\frac{2 \pi n}{c}\right)<t<\frac{1}{\pi} \operatorname{arccot}\left(-\frac{2 \pi(n+1)}{c}\right)\right\} .
\end{aligned}
$$

Assume $n \geq 0$. Since $T^{\prime}$ is increasing for $t \geq \frac{1}{2}$

$$
\text { inf } \begin{aligned}
\left\{T^{\prime}(t): t \in I_{n}\right\} & =\frac{c}{2} \csc ^{2}\left(\operatorname{arccot}\left(-\frac{2 \pi n}{c}\right)\right) \\
& =\frac{c^{2}+4 \pi^{2} n^{2}}{2 c}
\end{aligned}
$$

and

$$
m\left(I_{n}\right) \inf \left\{T^{\prime}(t): t \in I_{n}\right\}=\frac{c^{2}+4 \pi^{2} n^{2}}{2 \pi c} \arctan \left(\frac{2 \pi c}{c^{2}+4 \pi^{2} n(n+1)}\right)
$$

Thus

$$
\lim _{n \rightarrow \infty} m\left(I_{n}\right) \inf \left\{T^{\prime}(t): t \in I_{n}\right\}=1 .
$$

A similar calculation shows that

$$
\lim _{n \rightarrow-\infty} m\left(I_{n}\right) \inf \left\{T^{\prime}(t): t \in I_{n}\right\}=1,
$$

so there is $K>0$ such that

$$
m\left(I_{n}\right) \inf \left\{T^{\prime}(t): t \in I_{n}\right\} \geq K \text { for all } n .
$$

Carrying Lebesgue measure to $\partial \mathcal{O}$ with the exponential map gives

$$
\lambda\left(A_{n}\right) \text { inf }\left\{\left|f^{\prime}(\zeta)\right|: \zeta \in A_{n}\right\} \geq K,
$$

and $f$ satisfies condition (ii) of Theorem 3.1. 
Since $f$ is a singular inner function in $\mathscr{F}, \log \left|f^{\prime}\left(e^{i \theta}\right)\right| \in L^{1}$ and from Theorem 3.1 we have that

$$
h_{\mu}(f)=\frac{1}{2 \pi} \int_{-\pi}^{\pi} P_{\delta}(\theta) \log \left|f^{\prime}\left(e^{\mathrm{i} \theta}\right)\right| d \theta
$$

Now

$$
f^{\prime}(z)=\frac{-2 c \exp \left\{-c \frac{1+z}{1-z}\right\}}{(1-z)^{2}}
$$

so that

$$
\lim _{r \rightarrow 1} f^{\prime}\left(r e^{\mathrm{i} \theta}\right)= \begin{cases}\frac{-2 c \exp \left\{-c \frac{1+e^{\mathrm{i} \theta}}{\left.1-e^{i \theta}\right\}}\right.}{\left(1-e^{\mathrm{i} \theta}\right)^{2}} & \text { if } \theta \neq 0 \\ 0 & \text { if } \theta=0\end{cases}
$$

and

$$
\left|f^{\prime}\left(e^{\mathrm{i} \theta}\right)\right|=\frac{2 c}{\left|1-e^{\mathrm{i} \theta}\right|^{2}} \quad \text { if } \theta \neq 0
$$

However,

$$
\begin{aligned}
\log \left|f^{\prime}(z)\right| & =\log \frac{2 c}{|1-z|^{2}}-c \operatorname{Re} \frac{1+z}{1-z} \\
& =\frac{1}{2 \pi} \int_{-\pi}^{\pi} P_{z}\left(e^{\mathrm{i} \theta}\right) \log \left|f^{\prime}\left(e^{\mathrm{i} \theta}\right)\right| d \theta-c \operatorname{Re} \frac{1+z}{1-z}
\end{aligned}
$$

Thus

$$
\begin{aligned}
h_{\mu}(f) & =\log \left|f^{\prime}(\delta)\right|+c \operatorname{Re} \frac{1+\delta}{1-\delta} \\
& =\log \frac{2 c}{(1-\delta)^{2}} .
\end{aligned}
$$

Since $\delta$ is the unique real solution of $f(z)=z$ such that $0<\delta<1,-c(1+\delta)=$ $(1-\delta) \log \delta$ and

$$
c=\frac{1-\delta}{1+\delta} \log \frac{1}{\delta}
$$

so that

$$
h_{\mu}(f)=\log \left(\frac{1}{1-\delta^{2}} \log \frac{1}{\delta^{2}}\right)
$$

Since

$$
\left|f^{\prime}\left(e^{\mathrm{i} \theta}\right)\right|=\frac{c}{1-\cos \theta}, \inf \left\{\left|f^{\prime}\left(e^{\mathrm{i} \theta}\right)\right|\right\}=c / 2,
$$

and Theorem 2.2, with Remark 2.4 shows that $f$ is a one-sided Bernoulli shift if $c>2$. 
Notice that if we parametrize the family

$$
\left\{\exp \left[-c \frac{1+z}{1-z}\right]: c>0\right\}
$$

in terms of the Denjoy-Wolff points $\delta$ we have the family

$$
\left\{\delta^{[(1-\delta) /(1+\delta)](1+z) /(1-z)]}: 0<\delta<1\right\}
$$

of inner functions whose entropy is given by

$$
h_{\delta}=\log \left(\frac{1}{1-\delta^{2}} \log \frac{1}{\delta^{2}}\right)
$$

Since $h_{\delta}$ is increasing on $0<\delta<1$, no two functions in this family are isomorphic.

\section{Rigidity of inner functions}

In this section we use the technique of Shub and Sullivan [20] to show that metrically isomorphic inner functions with finitely many singularities on $\partial \mathbb{O}$ and Denjoy-Wolff points in $\mathbb{O}$ are diffeomorphic to within a rotation.

THEOREM 5.1. Let $f$ and $g$ be in $\mathscr{F}$ with $f(0)=g(0)=0$ and with finite nonempty sets $\mathbb{E}_{f}$ and $\mathbb{E}_{g}$ respectively of singularities on $\partial \mathrm{O}$. If $f^{*}$ and $g^{*}$ are measure theoretically isomorphic on $(\partial \mathbb{O}, \lambda)$ there exists an isometry $\psi$ of $\mathbb{O}$ and a constant $c$ of modulus one such that $\psi^{-1} \circ g \circ \psi=c f$.

Proof. Since $f(0)=g(0)=0, f^{*}$ and $g^{*}$ preserve Lebesgue measure on 20 . Assume $\varphi \circ f=g \circ \varphi$ on $\partial \mathbb{O}$ where $\varphi$ is a metric isomorphism of $(\partial \mathbb{Q}, \lambda)$. Then $\varphi \circ f=g \circ \varphi$ on $\partial \mathbb{O}-\mathbb{E}_{f} \cup \mathbb{E}_{g}$, and if $J_{f}$ and $J_{g}$ denote the Jacobian of $f$ and $g$ respectively on $\partial \mathbb{Q}$, $J_{f}=J_{g} \circ \varphi$ a.e. on $\partial \mathbb{O}$. However, $\left|f^{\prime}\right|=J_{f}$ and $\left|g^{\prime}\right|=J_{g}$ a.e. on $\partial \mathbb{O}-\mathbb{E}_{f} \cup \mathbb{E}_{g}$ so $\left|f^{\prime}\right|=\left|g^{\prime}\right| \circ \varphi$ a.e. on $\partial \mathbb{O}-\mathbb{E}_{f} \cup \mathbb{E}_{g}$.

Let $A$ be an arc in $\partial \mathbb{O}-\mathbb{E}_{g}$. Since $\left|g^{\prime}\right|$ is real analytic on $A$, there is an arc $J \subset A$ such that $\left|g^{\prime}\right|$ is monotone on $J$. Define $I=\left|g^{\prime}\right|(J)$ and let $h: I \rightarrow J$ be such that $h \circ\left|g^{\prime}\right|=i d$ on $J$.

Let $B_{k}$ denote the arcs between the singularities of $f$ on 20 . Since $\varphi^{-1}(J)$ has positive Lebesgue measure, there is some $k$ such that $\varphi^{-1}(J) \cap B_{k}$ has positive measure. If $x \in B_{k} \cap \varphi^{-1}(J)$ and $x \notin \mathbb{E}_{g}$, then $\left|f^{\prime}\right|(x)=\left|g^{\prime}\right| \circ \varphi(x) \in I$ and $\left|f^{\prime}\right|^{-1}(I) \cap$ $\left(B_{k} \cap \varphi^{-1}(J)\right)$ is not empty. Since $\left|f^{\prime}\right|^{-1}(I)$ is a countable union of arcs, there exists an arc $K \subset\left|f^{\prime}\right|^{-1}(I)$ such that $K \cap\left(B_{k} \cap \varphi^{-1}(J)\right)$ has positive measure.

On $K \cap\left(B_{k} \cap \varphi^{-1}(J)\right)-\mathbb{E}_{g}, h \circ\left(\left|g^{\prime}\right| \circ \varphi\right)=\left(h \circ|g|^{\prime}\right) \circ \varphi=\varphi$. Since $\left|g^{\prime}\right| \circ \varphi=\left|f^{\prime}\right|$ on $K \cap B_{k}-\mathbb{E}_{g}, h \circ\left|f^{\prime}\right|=\varphi$ a.e. on $K \cap B_{k}-\mathbb{E}_{\xi}$, and there is some arc $L \subset K \cap b_{k}$ such that $\varphi=h \circ\left|f^{\prime}\right|$ a.e. on $L$.

Since $\varphi$ has Radon Nikodym derivative one a.e. on $L$ and $h \circ\left|f^{\prime}\right|$ is real analytic on $L$, the derivative of $h \circ\left|f^{\prime}\right|$ is one everywhere on $L$ and $h \circ\left|f^{\prime}\right|$ is an isometry on $L$. Let $\psi$ denote this isometry extended to $\mathbb{O}$. We have $\left|f^{\prime}\right|=\left|g^{\prime}\right| \circ \psi$ a.e. on $L$ and hence everywhere on $L$ by continuity.

Let $\tilde{g}=\psi^{-1} \circ g \circ \psi$ so that $\left|\tilde{g}^{\prime}\right|=\left|\tilde{g}^{\prime}\right| \circ \psi=\left|f^{\prime}\right|$ on $L$. Since

$$
\frac{z \tilde{g}^{\prime}(z)}{\tilde{g}(z)}=\left|\tilde{g}^{\prime}(z)\right|=\left|f^{\prime}(z)\right|=\frac{z f^{\prime}(z)}{f(z)}
$$

for $z \in L$ it follows that $(\log \tilde{g}(z))^{\prime}=(\log f(z))^{\prime}$ on $L$. Thus $\tilde{g}(z)=c f(z)$ for all $z \in L$. 
Since $|f(z)|=|\tilde{g}(z)|=1$ on $L,|c|=1$. Since $\tilde{g}$ and $c f$ are analytic on $\mathbb{O} \cup L$, it follows that $\tilde{g}=\psi^{-1} \circ g \circ \psi=c f$ on $\mathbb{O}$.

Corollary 5.2. Let $f$ and $g$ be in $\mathscr{F}$. Assume the Denjoy-Wolff points, $\delta_{1}$ and $\delta_{2}$, of $f$ and $g$ are in $\mathbb{O}$. If $\left(\partial \mathbb{O}, \mu_{\delta_{1}}, f^{*}\right)$ and $\left(\mathbb{O}, \mu_{\delta_{2}}, g^{*}\right)$ are metrically isomorphic, where $\mu_{\delta_{1}}, \mu_{\delta_{2}}$ are the invariant measures given by (4) of $\S 1$, there exist Möbius transformations $\psi_{1}$ and $\psi_{2}$ and a constant $c$ of modulus one such that

$$
\psi_{2}^{-1} \circ g \circ \psi_{2}=c\left(\psi_{1}^{-1} \circ f \circ \psi_{1}\right) \text { on } \mathbb{O} \text {. }
$$

Proof. Let $\psi_{1}$ and $\psi_{2}$ denote the Möbius transformations which send $\mathbb{O}$ to $\mathbb{O}, \partial \mathbb{O}$ to $\partial \mathrm{O}$ and $\delta_{1}$ and $\delta_{2}$ respectively to zero. The conjugates $\psi_{1}^{-1} \circ f \circ \psi$ and $\psi_{2}^{-1} \circ g \circ \psi_{2}$ fix zero and the corollary follows.

\section{REFERENCES}

1. Jon Aaronson. Ergodic theory of inner functions of the upper half plane. Ann. Inst. Henri Poincairé 14 (1978), 233-253.

2. Jon Aaronson. A remark on exactness of inner functions. J. London Math. Soc. (2)23 (1981), 469-474.

3. P. R. Ahern \& D. N. Clark. On inner functions with $H^{P}$-derivative. Michigan Math. J. 21 (1974), 115-127.

4. R. B. Burckel. Iterating analytic self maps of discs. Amer. Math. Monthly 88 (1981), 387-460.

5. C. Caratheodory. Theory of Functions. Vol. II. Chelsea: New York, 1954.

6. M. R. Cullen. Derivatives of singular inner functions. Michigan Math. J. 18 (1971), 283-287.

7. J. L. Fernández. A note on entropy and inner functions. Israel J. Math. 53 (1986), 158-162.

8. F. Forelli. The isometries of $H^{p}$. Canad. J. Math. 16 (1964), 721-728.

9. J. B. Garnett. Bounded Analytic Functions. Academic Press: New York, 1981.

10. G. Letac. Which functions preserve Cauchy laws? Proc. AMS 67 (1977), 277-286.

11. N. F. G. Martin. On finite Blaschke products whose restrictions to the unit circle are exact endomorphisms. Bull. London Math. Soc. 15 (1983), 343-348.

12. N. F. G. Martin. Classification of some finite Blaschke products as metric endomorphisms. J. Math. Anal. Appl. 114 (1986), 205-209.

13. N. F. G. Martin and J. W. England. Mathematical theory of entropy. Encyl. of Math. and Appl. Vol. 12. Addison-Wesley: New York, 1981.

14. J. H. Neuwirth. Ergodicity of some mappings of the circle and the line. Israel J. Math. 31 (1978), 359-367.

15. W. Parry. Entropy and generators in ergodic theory. Math. Lecture Note Series. Benjamin; New York, 1969.

16. B. Petit. Theorie ergodic: Classification de certaines transformation réeles. Ann. Inst. Henri Poincairé 15 (1979), 25-32.

17. C. Pommerenke. On ergodic properties of inner functions. Math. Ann. 256 (1981), 43-50.

18. W. Rudin. Real and Complex Analysis. 3rd Ed. McGraw-Hill: New York, 1987.

19. M. Rychlik. Bounded variation and invariant measures. Studia Math. 86 (1983), 69-80.

20. M. Shub \& D. Sullivan. Expanding endomorphisms of the circle revisited. Ergod. Th. \& Dynam. Sys. 5 (1985), 285-289.

21. J. Wolff. Sur une généralisation d'un théorème de Schwartz. C. R. Acad. Sci. Paris 182 (1926), 918-920 and 183 (1926), 500-502. 